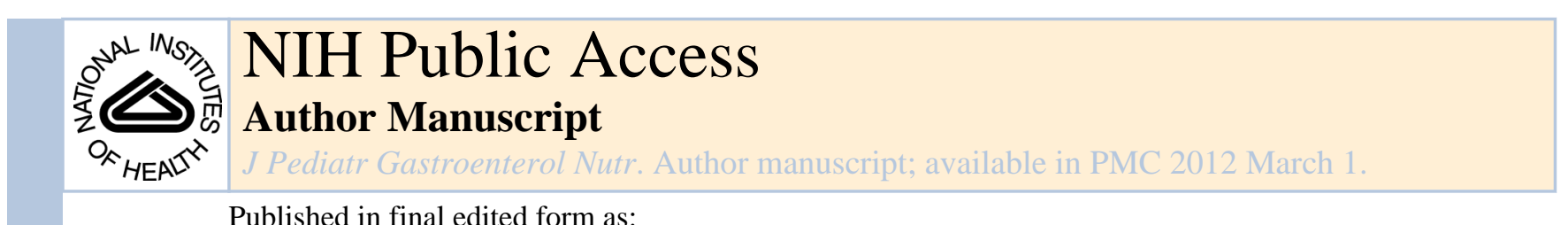

J Pediatr Gastroenterol Nutr. 2011 March ; 52(3): 295-299. doi:10.1097/MPG.0b013e3181f5714e.

\title{
Patient and Parent Psychosocial Factors Associated with Health-Related Quality of Life in Pediatric Inflammatory Bowel Disease
}

Michele Herzer, Ph.D. ${ }^{1}$, Lee A. Denson, M.D. ${ }^{1,2}$, Robert N. Baldassano, M.D. ${ }^{3,4}$, and Kevin A. Hommel, Ph.D. ${ }^{1,2}$

${ }^{1}$ Cincinnati Children's Hospital Medical Center

${ }^{2}$ University of Cincinnati College of Medicine

${ }^{3}$ Children's Hospital of Philadelphia

${ }^{4}$ University of Pennsylvania School of Medicine

\begin{abstract}
Objectives-To examine the mediating role of youth depressive symptoms in the relationship between parent distress and youth health-related quality of life (HRQOL) in a sample of adolescents with inflammatory bowel disease (IBD).

Methods-Sixty-two adolescents, aged 13-17 years, with a confirmed diagnosis of IBD completed assessments of depressive symptoms and HRQOL. Each adolescent's primary caregiver completed a measure of parent stress related to their child's illness. Pediatric gastroenterologists provided data for disease severity assessments.
\end{abstract}

Results-Multiple regression analyses revealed that adolescent depressive symptoms fully mediated the relationship between parent distress and several dimensions of HRQOL (i.e., General Well-Being, Emotional Functioning, Social Functioning, and Total HRQOL). Moreover, mediation was observed after statistically controlling for the impact of disease severity, IBD diagnosis, and significant demographic parameters on HRQOL.

Conclusions-Findings suggests that adolescent depressive symptoms may serve as the mechanism through which parent distress is linked to poorer HRQOL in adolescents with IBD. Close monitoring of parent illness-related distress and adolescent depressive symptoms as well as clinical interventions targeting these factors, are needed to promote optimal outcomes in adolescents with IBD.

\section{Keywords}

Inflammatory Bowel Disease; Adolescents; Quality of Life; Parent Distress; Depression

\footnotetext{
Corresponding Author: Kevin A. Hommel, Ph.D., Cincinnati Children's Hospital Medical Center, Division of Behavioral Medicine and Clinical Psychology, Center for Adherence and Self Management, 3333 Burnet Ave. MLC 7039, Cincinnati, OH 45229, Office: 513-803-0407; Fax: 513-803-0415, kevin.hommel@cchmc.org.

Disclosure: There is no conflict of interest to declare.

This is a PDF file of an unedited manuscript that has been accepted for publication. As a service to our customers we are providing this early version of the manuscript. The manuscript will undergo copyediting, typesetting, and review of the resulting proof before it is published in its final citable form. Please note that during the production process errors may be discovered which could affect the content, and all legal disclaimers that apply to the journal pertain.
} 
The diagnosis of inflammatory bowel disease (IBD) can be emotionally challenging for both children and parents. IBD involves unpleasant symptoms (e.g., diarrhea, abdominal pain) and treatment regimens that are complex and time-consuming (e.g., daily medication, surgical procedures). Disease symptoms and medication side effects can make IBD an embarrassing illness during childhood and adolescence and, consequently, psychosocial functioning and health-related quality of life (HRQOL) may be impaired. Indeed, research demonstrates that among children and adolescents with IBD, body image (e.g., short stature and weight gain), physical symptoms (e.g., diarrhea), and frequent visits to the bathroom are primary concerns [1-3], and adolescents experience lower HRQOL in physical functioning, psychological functioning, and autonomy domains [4-6]. While disease severity and level of parent stress have been identified as predictors of lower HRQOL in youth with other chronic conditions, there is very limited understanding of risk factors, other than disease severity, associated with poorer HRQOL in IBD. Since the focus of health care in pediatrics has shifted from concentrating predominantly on mortality and morbidity to a more comprehensive view that encompasses patient-reported physical functioning as well as psychosocial functioning [7], HRQOL has become an important outcome variable for medical providers [8]. It is therefore important to identify factors that contribute to poor HRQOL in adolescents with IBD.

Poor HRQOL among youth with chronic medical conditions has been linked to greater psychosocial and internalizing (i.e., emotional and mood difficulties such as anxiety and depression) problems. For example, among youth with epilepsy [9], obesity [10], asthma [11], and diabetes [12], depressive symptoms and psychosocial functioning have been identified as strong predictors of poor quality of life, suggesting that internalizing difficulties may be an important contributing factor to HRQOL. In children and adolescents with IBD, research has shown a tendency for this population to experience elevated rates of internalizing difficulties, particularly anxiety and depression, compared to otherwise healthy youth [13]. While the etiology of depression in this population may have both a psychological (e.g., difficulties adjusting and/or coping with the chronic demands of IBD) and biological (e.g., inflammatory proteins) basis, recent research indicates that adolescents with IBD are 4.6 times more likely to experience significant symptoms of anxiety and depression compared to healthy adolescents [14]. However, more current and population based data are needed to confirm this finding. Nevertheless, patient emotional distress may be an important factor to consider when assessing HRQOL among children and adolescents with IBD.

Similar to patients' experience, parents of children and adolescents with IBD exhibit heightened levels of emotional distress and poorer functioning [15]. Poor psychosocial functioning among mothers in youth with IBD has been correlated with greater adolescent depressive symptoms and more negative IBD health outcomes [16], In a separate study, maternal functioning was related to poorer HRQOL in adolescents with IBD [17]. Yet, no research has examined these variables together to determine underlying mechanisms through which they are related despite the theoretical underpinnings of the findings, which are congruent with Thompson's Transactional Stress and Coping model [18]. This model has been empirically tested in various pediatric populations, and posits that in the context of a child's chronic medical condition, the parent's own functioning plays a key role in the child's psychological adjustment along with sociodemographic and illness parameters (e.g., diagnosis, prognosis), and child factors (e.g., cognitive appraisal). For example, Janicke et al. [19] found that increased parent distress was associated with poorer child-reported HRQOL in a sample of overweight youth, and that this relationship was mediated by child depressive symptoms. Parent distress may similarly be a key factor in understanding HRQOL among youth with IBD. 
It is clear that HRQOL among children and adolescents with IBD is an important outcome variable to consider. However, little is currently known about the psychosocial risk factors that contribute to low HRQOL in this population. Given the relationship between parent distress and adolescent HRQOL as well as research documenting elevated levels of internalizing difficulties in youth with IBD, it is plausible that adolescent HRQOL is impacted by both their own level of distress as well as their parents'. This study was designed to examine the link between parent distress, adolescent depressive symptoms, and adolescent HRQOL in a sample of adolescents with IBD. Specifically, the mediating role of youth depressive symptoms in the relationship between parent distress and youth HRQOL was tested. We hypothesized that child depressive symptoms would mediate the relationship between parent distress and HRQOL.

\section{Materials and Methods}

\section{Participants}

Participants included 62 adolescents aged 13-17 years with a confirmed diagnosis of IBD and a prescribed treatment regimen of 5-aminosalycylic acid (5-ASA) and/or 6-MP/ azathioprine. Each adolescent's primary caregiver also participated in the study. Exclusion criteria included patients with a diagnosis of a neurocognitive disorder, prescription of $>1$ $\mathrm{mg} / \mathrm{kg} /$ day corticosteroid (due to increased likelihood of psychiatric side effects[20-21]), comorbid chronic illness diagnosis, and lack of English fluency. One hundred six patients were eligible for the study; of these, 83 were able to be contacted for recruitment, 13 declined participation, and 8 did not provide complete data. Primary reasons for declining participation included blood draw requirement, time constraints, and/or lack of interest. Thus, a final sample of 62 adolescents ( 35 male, 27 female) receiving treatment for IBD (CD $n=49$; UC $n=13$ ) and their caregivers participated in the current study. The majority of adolescents were Caucasian $(88.7 \%)$ and had a mean age of 15.4 years $(S D=1.4)$. Patient and caregiver characteristics are outlined in Table 1. Sample characteristics were generally representative of the pediatric IBD population and recently published studies in IBD [22].

\section{Procedure}

Study recruitment occurred at two large pediatric medical centers in the midwest $(n=28)$ and northeast $(n=34)$ United States. Eligibility was determined by study personnel via chart review. Eligible families were contacted during regularly scheduled gastroenterology clinic appointments, scheduled Infliximab infusions, or via telephone. Informed consent/assent were obtained from both the adolescents and their caregivers, after first confirming study eligibility with caregivers. Disease severity assessments were completed by study personnel using data provided by each participant's gastroenterologist for the clinic appointment corresponding to the study visit or the most recent clinic appointment. Participants were compensated $\$ 25$ for participation. Institutional Review Boards at each hospital approved this study.

\section{Measures}

Demographic Questionnaire-A demographic questionnaire assessing family characteristics (e.g., household income, marital status, parental education) was completed by parents.

Pediatric Crohn's Disease Activity Index (PCDAl; [23])—The well-validated PCDAI [23] assesses Crohn's disease activity using both subjective (e.g., pain) and objective criteria (e.g., physical exam), laboratory findings, and growth parameters. Scores range from 0-100: $\leq 10=$ inactive disease; $11-30=$ mild disease, and $>30=$ moderate-tosevere disease activity[24]. Internal consistency was .95 in this sample. 
Lichtiger Colitis Activity Index (LCAI; [25])—The LCAI [25] uses both subjective and objective criteria to assess 8 UC symptoms (score 0-21): daily stool frequency, nocturnal diarrhea, visible blood in stool, fecal incontinence, abdominal pain or cramping, general well-being, abdominal tenderness, and need for anti-diarrheal medication, with higher scores representing more severe disease. LCAI scores range from $0-21$. Scores $\leq 2$ indicate quiescent disease; $<10$ indicate a response to therapy; $\geq 10$ indicate active disease and no response to therapy [26]. Internal consistency was .85 in this sample.

Pediatric Inventory for Parents (PIP; [27])—The PIP is a 42-item parent-report questionnaire using 5-point Likert scaling that measures both the frequency (PIP-F) and difficulty (PIP-D) of illness-related parent stress across 4 factors: Communication, Medical Care, Role Functioning, and Emotional Distress. Good validity and internal consistency has been demonstrated previously ( $\alpha=.80-.96)$ [27] and also within the current study (PIP-F, $\alpha$ $=.96 ;$ PIP-D,$\alpha=.95$ ).

Children's Depression Inventory (CDI; [28])—The CDI is a well-validated 27-item self-report questionnaire of depressive symptomatology. Using a 3-point Likert scale that ranges from 0 (no symptom) to 2 (distinct symptom), the CDI assesses negative mood, interpersonal problems, ineffectiveness, anhedonia, and negative self-esteem. The CDI total scores ranges from 0 to 54 with a clinical cutoff score of 13 or higher indicative of elevated depressive symptoms. A high degree of internal consistency was demonstrated within the current sample (coefficient $\alpha=0.90$ ).

IMPACT-III [29]: The IMPACT-III is a 35-item self-report, IBD-specific measure of HRQOL. It assesses the extent to which an adolescent is affected by a particular issue (e.g., stomach pain, missing out on certain activities) using 5-point Likert scaling, with lower scores indicating poorer HRQOL. Originally, 6 domains (i.e., bowel symptoms, systemic symptoms, social/functional concerns, body image, test and treatment concerns, and emotional concerns) were proposed. However, a recent examination of this measure's factor structure revealed 4 factors with good to excellent reliability: general well-being, emotional functioning, social functioning, and body image [30]. This factor structure was used in this study, along with a total score of HRQOL created by adding all 35 items. This total score demonstrated excellent internal consistency for the current sample (Cronbach's $\alpha=.95$ ).

Statistical Analyses-Descriptive statistics were calculated including means and standard deviations. Bivariate correlations were conducted to identify significant relationships between continuous demographic or medical characteristics (i.e., adolescent age, disease severity) and IMPACT-III scores of adolescent HRQOL. Also, to identify significant differences of demographic/medical characteristics on IMPACT-III scores, t-tests were conducted on dichotomous variables (i.e., adolescent gender, IBD diagnosis) and oneway analyses of variance (ANOVA) were conducted on the remaining categorical variables (i.e., adolescent grade, adolescent ethnicity, parent marital status, parent education level, parent employment status, household income). Any significant demographic and/or medical variables were entered into subsequent analyses as covariates. Multiple regression analyses were performed to test mediation (i.e., parent stress $\rightarrow$ adolescent depressive symptomatology $\rightarrow$ adolescent HRQOL) using the Baron and Kenny framework [31]. The four factors of the IMPACT-III and the total score were used as outcome measures of adolescent HRQOL. Presence of mediation was determined by testing whether: 1) parent stress was associated with adolescent depressive symptoms, 2) parent stress was associated with dimensions of HRQOL, and 3) when parent stress and adolescent depressive symptoms were included in the same model, the effect of parent stress on HRQOL values was reduced. Standardized beta weights $(\beta)$ and significance levels $(p)$ are reported for relevant variables 
in each analysis. In all analyses, the Sobel test was used to detect statistical significance of the mediator [32]. Analyses were conducted in SPSS 15.0.1 (SPSS Inc., Chicago, IL).

\section{Results}

Preliminary analyses revealed a significant negative correlation between disease severity and each dimension of HRQOL (General Well-Being, $r=-.47, p<.01$; Emotional Functioning, $r=-.39, p<.01$; Social Functioning, $r=-.27, p<.05$; Body Image, $r=-.32$, $p=.01$; Total HRQOL, $r=-.45, p<.01)$. A significant difference of IBD diagnosis was also found on General Well-Being, $\mathrm{t}(59)=-2.91, p<.01$, Body Image, $\mathrm{t}(60)=-2.39, p<$. 01, and Total HRQOL, $\mathrm{t}(58)=-2.22, p<.01$, with adolescents diagnosed with Crohn's disease reporting lower HRQOL than those with ulcerative colitis. Body image was also found to be significantly different based on household income, $\mathrm{F}(8,49)=2.86, p=.01$, with adolescents from higher household income homes endorsing greater body image. Thus, disease severity, diagnosis, and income were significantly related to the primary outcome and were therefore entered into subsequent regression models simultaneously with the hypothesized predictors (i.e., parent stress and adolescent depressive symptoms). Additionally, adolescents had a mean CDI total depressive symptoms score of 5.85 (SD = $6.43)$; however, $15 \%$ of this sample endorsed symptoms falling in the clinical range ( $\geq 13)$.

Figure 1 provides a graphical representation of the mediator model. In the first step of each regression analysis (i.e., parent stress $\rightarrow$ adolescent depressive symptoms), parent stress predicted variance in adolescent depressive symptoms $(\beta=.42, p<.01)$. In the second step of each regression analysis (i.e., parent stress $\rightarrow$ adolescent HRQOL), parent stress significantly predicted variance in General Well-Being $(\beta=-.35, p<.01)$, Emotional Functioning $(\beta=-.31, p=.01)$, Social Functioning $(\beta=-.36, p<.01)$, and Total HRQOL $(\beta=-.37, p<.01)$. In the last step of each regression analysis (parent stress and adolescent depressive symptomatology $\rightarrow$ adolescent HRQOL), the effect of parent stress on adolescent General Well-Being $(\beta=-.09, p=$ n.s.; Sobel $=-3.04, p<.01)$, Emotional Functioning $(\beta=-.11, p=$ n.s.; Sobel $=-2.52, p=.01)$, Social Functioning $(\beta=-.13, p=$ n.s.; Sobel $=-2.87, p<.01)$, and Total HRQOL $(\beta=-.09, p=$ n.s.; Sobel $=-3.06, p<.01)$ became nonsignificant with adolescent depressive symptomatology also in the model. Thus, full mediation was found for all dimensions of HRQOL, with the exception of Body Image.

Given the possible overlap in items assessing emotional functioning between the CDI and the Emotional Functioning subscale of the IMPACT-III, a total HRQOL score was calculated with emotional functioning items removed. Identical regression analyses were conduced with this modified Total HRQOL variable. Parent stress significantly predicted adolescent depressive symptomatology $(\beta=.42, p<.01)$ and Total HRQOL $(\beta=-.36, p<$. 01 ), and with parent stress and adolescent depressive symptomatology in the model, parent stress was no longer a significant predictor of Total HRQOL $(\beta=-.07, p=$ n.s. $)$, indicating full mediation.

\section{Discussion}

This study is the first to examine the relationship of patient and parent psychosocial factors with HRQOL in adolescents with IBD. Results indicate that the mechanism through which parent distress is related to lower levels of HRQOL is adolescent depressive symptoms even after statistically controlling for disease severity and IBD diagnosis. Consistent with prior research, greater disease severity [3] as well as a diagnosis of Crohn's disease (rather than ulcerative colitis) [4] were associated with lower ratings across multiple HRQOL dimensions (e.g., body image, general well-being, and total HRQOL). Moreover, because disease severity, diagnosis, parenting stress, and adolescent depressive symptoms were entered into the model simultaneously, these findings suggest that relative to disease-related 
factors, parent and patient psychosocial factors may be stronger predictors of poorer HRQOL. However, these findings extend the literature by providing preliminary evidence of psychosocial factors that place this population at risk of experiencing greater disruptions in HRQOL.

Approximately $15 \%$ of these adolescents experienced depressive symptoms falling in the clinical range $(\geq 13)$, consistent with other IBD samples of older children and adolescents [33]. Depressive symptoms were directly related to lower perceived HRQOL in the areas of general well-being, emotional and social functioning, and body image. Moreover, adolescent depressive symptoms explained a significant proportion of the parent stress-adolescent HRQOL relationship across several dimensions of HRQOL, including general well-being, emotional functioning, social functioning, and overall HRQOL. This suggests that these adolescents are vulnerable to experiencing disruptions in daily functioning and well-being via two pathways; first, through their own emotional distress, and second, by being exposed to the adjustment difficulties experienced by their parents. The broader child development literature provides insight into the process by which parent and child functioning might interact to affect child psychosocial outcomes such as HRQOL. For example, research shows that children of distressed mothers are at increased risk of internalizing and externalizing difficulties [34-35] via maladaptive parent-child interactions. Distressed parents have been found to provide inadequate support [36], be less responsive to the youth's needs [37], and grant less autonomy to the youth [38], consequently placing adolescents at elevated risk of experiencing greater emotional distress.

Data from the current study builds upon extant literature in several ways. To our knowledge, this is the first study to not only examine risk factors of poorer quality of life in pediatric IBD beyond disease severity, but also the contribution of caregiver psychosocial factors (i.e., parent distress) to the emotional functioning of youth with IBD. This is also an important first step towards articulating the complex relationships among patient and parent risk factors related to HRQOL in adolescents with IBD. The current study also used an empirically-validated theoretical framework (i.e., Thompson's transactional stress and coping model) to guide the hypotheses; which has been identified as a need in pediatric IBD research [39]. This study also suggests that the rate of clinically elevated symptoms of depression in this pediatric IBD sample is higher than that found in the general adolescent population [40].

These findings should be interpreted in the context of some limitations that carry important implications for future research. Characteristics of this particular sample (i.e., Caucasian, adolescents, predominantly inactive disease status, and mostly Crohn's disease diagnoses) may limit generalizability of findings to the broad IBD population. The high socioeconomic status (SES) of this sample, though similar to other published studies in pediatric IBD [41], must also be considered as a potential limitation to generalizability. In addition, since mothers comprised the majority of this sample, parental psychological distress predominantly reflects mothers' emotional distress. While fathers are widely underrepresented in pediatric research [42], and mothers are generally included as primary caregivers in pediatric IBD research, future research that gives greater focus on fathers' functioning will provide a more complete and accurate representation of how parents adjust to their child's IBD. This study also focused solely on adolescent depressive symptoms and did not examine other patient psychological variables such as anxiety and externalizing difficulties. Given the comorbidity between depression and other diagnoses such as anxiety in youth [43], future research should measure adolescent psychological functioning more broadly, thus capturing these interrelated psychological variables. It thus remains unclear whether parent stress is similarly related to other patient mood/behavioral states, and ultimately HRQOL, or whether adolescent depressive symptoms are uniquely more 
vulnerable to parent stress. Lastly, the cross-sectional nature of these analyses did not allow us to examine variations in parent stress and adolescent depressive symptoms over time or make causal inferences. Longitudinal research may identify critical time points in the course of IBD at which parents and adolescents are particularly vulnerable to experiencing difficulties and may also distinguish between chronic and reactive depression in adolescents.

Greater knowledge of factors that impede HRQOL in this population can guide clinical interventions by targeting such risk factors directly. The joint contribution of parent and adolescent distress to adolescent HRQOL highlights the need for family-based interventions that target adolescent and parent adjustment to IBD diagnosis. Parents may be key agents of change in their child's psychosocial functioning, and their involvement in clinical interventions may be crucial for improving the emotional well-being and HRQOL of adolescents with IBD. Such interventions may be most beneficial if they specifically address patient and parent emotional functioning, as well as the extent to which change in each individual's functioning is dependent on change in the other's. For example, clinical interventions may detect problematic levels of engagement between parents and adolescents (e.g., overprotective parenting, enmeshment) that may stem from parental distress and consequently affect adolescent functioning and HRQOL. Teaching adaptive coping and problem-solving skills, as well as effective communication and conflict resolution may be particularly useful in addressing these issues. Preliminary evidence in other pediatric populations suggests that these family-based treatment components lead to better outcomes [44].

\section{Acknowledgments}

Funding: This research was supported in part by NIDDK K23 DK079037, PHS Grant P30 DK 078392, Procter and Gamble Pharmaceuticals, Prometheus Laboratories, Inc., and Institutional Clinical and Translational Science Award NIH/NCRR Grant Number 1UL1RR026314.

\section{References}

1. Akobeng AK, et al. Quality of Life in Children with Crohn's Disease: A Pilot Study. J Pediatr Gastroenterol Nutr. 1999; 28((4)(Supplement)):S37-S39. [PubMed: 10204523]

2. Richardson G, et al. Quality of Life in Inflammatory Bowel Disease: A Cross-Cultural Comparison of English and Canadian Children. J Pediatr Gastroenterol Nutr. 2001; 32(5):573-578. [PubMed: 11429519]

3. Loonen HJ, et al. Measuring quality of life in children with inflammatory bowel disease: The Impact-II (NL). Qual Life Res. 2002; 11(1):47-56. [PubMed: 12003055]

4. Loonen $\mathrm{HJ}$, et al. Quality of life in paediatric inflammatory bowel disease measured by a generic and a disease-specific questionnaire. Acta Paediatr. 2002; 91(3):348-354. [PubMed: 12022311]

5. Cunningham $\mathrm{C}$, et al. Health-related quality of life in children and adolescents with inflammatory bowel disease. Child Health Care. 2007; 36(1):29-43.

6. De Boer M, et al. Health-related Quality of Life and Psychosocial Functioning of Adolescents with Inflammatory Bowel Disease. Inflamm Bowel Dis. 2005; 11(4):400-406. [PubMed: 15803032]

7. Sullivan M. The new subjective medicine: Taking the patient's point of view on healthcare and health. Soc Sci Med. 2003; 56:1595-1604. [PubMed: 12614708]

8. Hommel KA, Davis CM, Baldassano RN. Medication adherence and quality of life in pediatric inflammatory bowel disease. J Pediatr Psychol. 2008; 33(8):867-847. [PubMed: 18337262]

9. Orrin D, et al. Risk Factors for Poor Health-Related Quality of Life in Adolescents with Epilepsy. Epilepsia. 1999; 40(12):1715-1720. [PubMed: 10612334]

10. Zeller MH, Modi AC. Predictors of health-related quality of life in obese youth. Obesity (Silver Spring). 2006; 14(1):122-30. [PubMed: 16493130] 
11. Hommel KA, et al. Asthma-specific quality of life in older adolescents and young adults with longstanding asthma: The role of anxiety and depression. J Clin Psychol Med Settings. 2002; 9(3):185192.

12. Grey M, et al. Personal and family factors associated with quality of life in adolescents with diabetes. Diabetes Care. 1998; 21(6):909-914. [PubMed: 9614606]

13. Mackner LM, Crandall WV, Szigethy EM. Psychosocial functioning in pediatric inflammatory bowel disease. Pediatr. 2006; 12(3):239-244.

14. Mackner LM, Crandall WV. Brief Report: Psychosocial Adjustment in Adolescents with Inflammatory Bowel Disease. J Pediatr Psychol. 2006; 31(3):281-285. [PubMed: 15802606]

15. Engstrom I. Parental distress and social interaction in families with children with inflammatory bowel disease. J Am Acad Child Adolesc Psychiatry. 1991; 30:904-912. [PubMed: 1757439]

16. Tojek TM, et al. Maternal correlates of health status in adolescents with inflammatory bowel disease. J Psychosom Res. 2002; 52(3):173-179. [PubMed: 11897236]

17. MacPhee M, Hoffenberg EJ, Feranchak A. Quality-of-life factors in adolescent inflammatory bowel disease. Inflamm Bowel Dis. 1998; 4(1):6-11. [PubMed: 9552222]

18. Thompson RJ, et al. Psychological adjustment of children with cystic fibrosis: The role of child cognitive processes and maternal adjustment. J Pediatr Psychol. 1992; 17:741-755. [PubMed: 1484336]

19. Janicke DM, et al. Impact of Psychosocial Factors on Quality of Life in Overweight Youth. Obesity. 2007; 15(7):1799-1807. [PubMed: 17636099]

20. Kayani S, Shannon DC. Adverse behavioral effects of treatment for acute exacerbation of asthma in children: a comparison of two doses of oral steroids. Chest. 2002; 122(2):624-8. [PubMed: 12171842]

21. Soliday E, Grey S, Lande MB. Behavioral effects of corticosteroids in steroid-sensitive nephrotic syndrome. Pediatrics. 1999; 104(4):e51. [PubMed: 10506276]

22. Mackner LM, Crandall WV. Oral medication adherence in pediatric inflammatory bowel disease. Inflamm Bowel Dis. 2005; 11(11):1006-12. PMID: 16239847. [PubMed: 16239847]

23. Hyams JS, et al. Development and validation of a pediatric Crohn's disease activity index. J Pediatr Gastroenterol Nutr. 1991; 12(4):439-47. PMID: 1678008. [PubMed: 1678008]

24. Hyams J, et al. Evaluation of the Pediatric Crohn Disease Activity Index: A Prospective Multicenter Experience. Journal of Pediatric Gastroenterology and Nutrition. 2005; 41(4):416421. [PubMed: 16205508]

25. Lichtiger $\mathrm{S}$, et al. Cyclosporine in severe ulcerative colitis refractory to steroid therapy. N Engl J Med. 1994; 330(26):1841-5. PMID: 8196726. [PubMed: 8196726]

26. Fanjiang G, Russell GH, Katz AJ. Short- and Long-term Response to and Weaning from Infliximab Therapy in Pediatric Ulcerative Colitis. J Pediatr Gastroenterol Nutr. 2007; 44(3):312317. 10.1097/MPG.0b013e31802e98d4. [PubMed: 17325550]

27. Streisand R, et al. Childhood illness-related parenting stress: the pediatric inventory for parents. J Pediatr Psychol. 2001; 26(3):155-62. PMID: 11259517. [PubMed: 11259517]

28. Kovacs, M. The Children's Depression Inventory (CDI): Technical Manual. Multi-Health Systems; North Tonawanda, NY: 2003.

29. Otley A, et al. The IMPACT questionnaire: a valid measure of health-related quality of life in pediatric inflammatory bowel disease. J Pediatr Gastroenterol Nutr. 2002; 35(4):557-63. PMID: 12394384. [PubMed: 12394384]

30. Perrin JM, et al. Measuring quality of life in pediatric patients with inflammatory bowel disease: Psychometric and clinical characteristics. J Pediatr Gastroenterol Nutr. 2008; 46:164-171. [PubMed: 18223375]

31. Baron RM, Kenny DA. The moderator-mediator variable distinction in social psychological research: conceptual, strategic, and statistical considerations. J Pers Soc Psychol. 1986; 51(6): 1173-82. [PubMed: 3806354]

32. Holmbeck GN. Post-hoc probing of significant moderational and mediational effects in studies of pediatric populations. J Pediatr Psychol. 2002; 27(1):87-96. [PubMed: 11726683] 
33. Szigethy E, et al. Depressive Symptoms and Inflammatory Bowel Disease in Children and Adolescents: A Cross-Sectional Study. J Pediatr Gastroenterol Nutr. 2004; 39(4):395-403. [PubMed: 15448431]

34. Goodman SH, Gotlib IH. Risk for psychopathology in the children of depressed mothers: a developmental model for understanding mechanisms of transmission. Psychol Rev. 1999; 106(3): 458-90. [PubMed: 10467895]

35. Kim-Cohen J, et al. Maternal depression and children's antisocial behavior: nature and nurture effects. Arch Gen Psychiatry. 2005; 62(2):173-81. [PubMed: 15699294]

36. Lovejoy MC, et al. Maternal depression and parenting behavior: a meta-analytic review. Clin Psychol Rev. 2000; 20(5):561-92. [PubMed: 10860167]

37. Bush JP, et al. Mother-child patterns of coping with anticipatory medical stress. Health Psychology. 1986; 5:137-157. [PubMed: 3732228]

38. Rapee RM. Potential role of childrearing practices in the development of anxiety and depression. Clin Psychol Rev. 1997; 17(1):47-67. [PubMed: 9125367]

39. Mackner LM, Sisson DP, Crandall WV. Review: Psychosocial Issues in Pediatric Inflammatory Bowel Disease. J Pediatr Psychol. 2004; 29(4):243-257. [PubMed: 15148347]

40. Lewinsohn PM, et al. Major depression in community adolescents: Age at onset, episode duration, and time to recurrence. J Am Acad Child Adolesc Psychiatry. 1994; 33(6):809-818. [PubMed: 7598758]

41. Mackner LM, Crandall WV. Long-term psychosocial outcomes reported by children and adolescents with inflammatory bowel disease. Amer J Gastroenterol. 2005; 100(6):1386-1392. PMID: 15929775. [PubMed: 15929775]

42. Phares V, et al. Are Fathers Involved in Pediatric Psychology Research and Treatment? J. Pediatr. Psychol. 2005; 30(8):631-643. [PubMed: 15772363]

43. Brady EU, Kendall PC. Comorbidity of anxiety and depression in children and adolescents. Psychol Bull. 1992; 111(2):244-255. [PubMed: 1557475]

44. Wysocki T, et al. Effects of behavioral family systems therapy for diabetes on adolescents' family relationships, treatment adherence, and metabolic control. J Pediatr Psychol. 2006; 31(9):928-38. [PubMed: 16401678] 


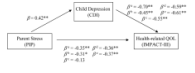

$+\ldots$

Figure 1.

Proposed mediator model: The mediating effect of adolescent depressive symptomatology on the parent distress-HRQOL link. 


\section{Table 1}

\section{Participant Characteristics}

\begin{tabular}{lc}
\hline$N$ & 62 \\
Adolescent age (years) & $15.4 \pm 1.4$ \\
Gender (\% female) & $43.5 \%$ \\
Ethnicity (\% White, not Hispanic origin) & $88.7 \%$ \\
Caregiver status & \\
$\quad$ Biological Mother (\%) & $85.5 \%$ \\
$\quad$ Biological Father (\%) & $9.7 \%$ \\
$\quad$ Adoptive Mother (\%) & $3.2 \%$ \\
$\quad$ Other (\%) & $1.6 \%$ \\
Caregiver marital status (\% married) & $88.7 \%$ \\
Caregiver education level (\% with at least a & $50.0 \%$ \\
college degree) & $86.3 \%$ \\
Caregiver employment status & $6.6 \pm 4.0$ \\
IB employed) & $79.0 \%$ \\
$\quad$ Crohn's Disease & $21.0 \%$ \\
$\quad$ Ulcerative Colitis & $9.83 \pm 9.75$ \\
Disease Severity & $11.68 \pm 10.0$ \\
PCDAI & $2.85 \pm 3.9$ \\
LCAI & $77.8 \pm 24.7$ \\
PIP Difficulty & $5.85 \pm 6.4$ \\
CDI (adolescent) & $144.55 \pm 19.9$ \\
IMPACT-III Total Score & $58.03 \pm 8.7$ \\
IMPACT-III General Well-Being & $46.15 \pm 7.1$ \\
IMPACT-III Emotional Functioning & $21.87 \pm 2.8$ \\
& $11.32 \pm 2.4$ \\
\hline
\end{tabular}

Note. Scores are shown as means \pm SD. PIP- Pediatric Inventory for Parents; CDI- Childhood Depression Inventory; PCDAI-Pediatric Crohn's Disease Activity Index; LCAI-Lichtiger Colitis Activity Index. 